\title{
Brecha digital, idioma inglés y su vínculo con la comprensión lectora en español
}

\author{
Digital Divide, English Language and Their Link with Spanish Reading \\ Comprehension
}

\section{Divisão digital, idioma inglês e seu vínculo com a compreensão de leitura em espanhol}

\author{
Hiram Herrera Rivas \\ Universidad Politécnica de Victoria, México \\ hiramhr@gmail.com \\ https://orcid.org/0000-0002-2650-8932 \\ Ramón Ventura Roque Hernández \\ Universidad Autónoma de Tamaulipas, México \\ rvhernandez@uat.edu.mx \\ https://orcid.org/0000-0001-9727-2608
}

\section{Resumen}

Para toda universidad es de gran importancia la detección oportuna de factores de éxito académico con la finalidad de generar estrategias adecuadas de tutoría que contribuyan con la finalización de estudios en tiempo y forma. El objetivo de esta investigación fue profundizar en el entendimiento de la primera brecha digital, el dominio del idioma inglés y la relación de ambos con la comprensión lectora en español de los alumnos de nuevo ingreso en la Universidad Politécnica de Victoria (UPV). Se utilizó un diseño de investigación cuantitativo, relacional y retrospectivo, en donde se analizaron los resultados del Examen Ceneval Exani-II que se aplicó a los alumnos de nuevo ingreso de dicha institución, ubicada en Tamaulipas, México, en los años 2015, 2016 y 2017. 


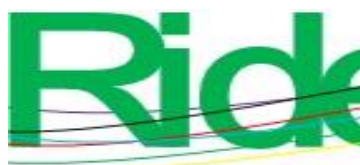

Revista Iberoamericana para la
Investigación y el Desarrollo Educativo
ISSN $2007-7467$

Se realizó un análisis unifactorial con cuatro grupos de estudio creados a partir de los datos. Esto permitió identificar una tendencia de aumento de la comprensión lectora ante la presencia del acceso a internet en casa y de un dictamen favorable del idioma inglés. En una segunda fase, se procedió con un análisis con dos factores con el que se pudo observar cada variable de manera independiente y la interacción de ambas. A través de las pruebas se pudo demostrar estadísticamente en todos los años que el dominio del idioma inglés incide positivamente en la comprensión lectora en español (pruebas Scheirer-Ray-Hare, $p=0.0$ ). También se observó una tendencia favorable en la comprensión lectora en español en los alumnos que tienen internet en casa (año 2017, prueba Scheirer-Ray-Hare, $p=0.0$ ). De esta manera, los alumnos que tienen internet en casa y que obtuvieron un dictamen satisfactorio en el idioma inglés obtuvieron las puntuaciones más altas, mientras que los alumnos sin este servicio en casa y con un dictamen insatisfactorio en inglés tuvieron las puntuaciones más bajas (pruebas Kruskal-Wallis, $p=0.0$ ). Estos resultados muestran que existen estudiantes de nuevo ingreso en la UPV que inician sus estudios universitarios en desigualdad de condiciones con los demás. Es preocupante que aún hoy en día existan estudiantes sin acceso a internet en casa y sin un dominio satisfactorio la lengua inglesa, pues son condiciones que reducen sus probabilidades de éxito académico. Es necesario reflexionar sobre la desigualdad que afecta a estos alumnos y las maneras en que se puede mejorar esta situación desde el interior de la misma universidad.

Palabras clave: brecha digital, comprensión, educación superior, idioma inglés, lectura.

\section{Abstract}

It is important that every university timely detect academic success factors that contribute students to finish their studies in a timely manner so a correct tutoring strategy can be applied. The objective of this research was to deepen the knowledge of digital gap, English language and the relationship of both with the reading comprehension of new students entering the Polytechnic University of Victoria (UPV). A quantitative, relational and retrospective research design was used to analyze the results of the Ceneval Exani-II Exam that was applied to new students of said institution, located in Tamaulipas, Mexico, for years 2015, 2016 and 2017. 


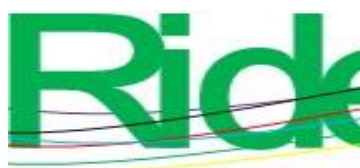

Revista Iberoamericana para la Investigación y el Desarrollo Educativo ISSN $2007-7467$

First, a one-factor analysis was made with four study groups created in the data; this allowed identifying a tendency to increase reading comprehension in the presence of Internet access at home and a favorable grade in English language. In a second phase, we proceeded with a two-factor analysis with which we could observe each variable independently and the interaction of both. By means of the tests it was possible to statistically demonstrate in all the years that English language proficiency has a positive effect on the reading comprehension in Spanish (Scheirer-Ray-Hare tests, $\mathrm{p}=0.0$ ). A favorable trend in Spanish reading comprehension was observed in students who have Internet at home (year 2017, ScheirerRay-Hare tests, $\mathrm{p}=0.0$ ). Thus, students who have Internet at home and who obtained a satisfactory grade in English language obtained the highest scores, while students without Internet at home and with an unsatisfactory grade in English had the lowest scores (KruskalWallis tests, $\mathrm{p}=0.0$ ). These results indicate that there are new students at the UPV who begin their university studies in unequal conditions with others. It is worrisome that even today there are students without internet access at home and without a satisfactory proficiency in English language, as these are conditions that reduce their chances of academic success. It is necessary to reflect on the inequality affecting these students and the ways in which this situation can be improved from within the university.

Keywords: digital divide, comprehension, higher education, English language, reading.

\section{Resumo}

Para cada universidade, a detecção oportuna de fatores de sucesso acadêmico é de grande importância, a fim de gerar estratégias de tutoria adequadas que contribuam para a conclusão dos estudos em tempo hábil. O objetivo desta pesquisa foi aprofundar a compreensão do primeiro fosso digital, a proficiência na língua inglesa e a relação de ambos com a compreensão de leitura em espanhol de novos alunos da Universidade Politécnica de Victoria (UPV). Utilizou-se um projeto de pesquisa quantitativa, relacional e retrospectiva, que analisou os resultados do Exame Ceneval Exani-II aplicado aos novos alunos da referida instituição, localizada em Tamaulipas, México, nos anos de 2015, 2016 e 2017.

Uma análise unifatorial foi realizada com quatro grupos de estudo criados a partir dos dados. Isso nos permitiu identificar uma tendência a aumentar a compreensão de leitura na presença de acesso à Internet em casa e uma opinião favorável do idioma inglês. Numa segunda fase, procedeu-se a uma análise com dois fatores com os quais cada variável pôde ser observada 


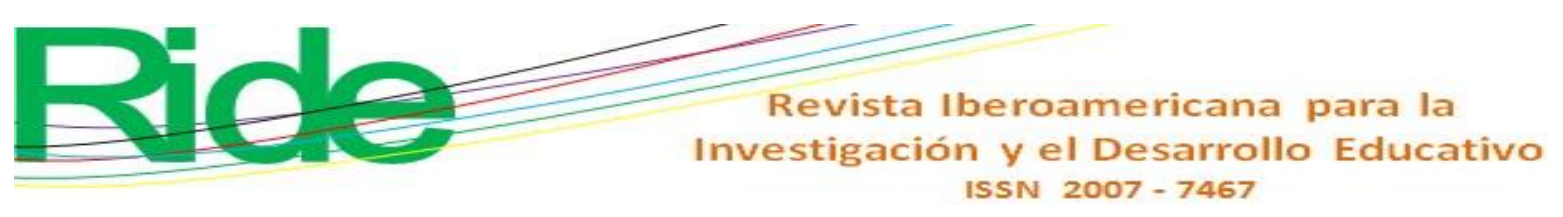

independentemente e a interação de ambos. Através dos testes, foi possível demonstrar estatisticamente em todos os anos que a proficiência em inglês tem um impacto positivo na compreensão da leitura em espanhol (testes de Scheirer-Ray-Hare, $\mathrm{p}=0,0$ ). Também foi observada uma tendência favorável à compreensão da leitura em espanhol em estudantes que possuem internet em casa (2017, teste Scheirer-Ray-Hare, $p=0,0)$. Dessa forma, os alunos que possuem internet em casa e obtiveram uma opinião satisfatória no idioma inglês obtiveram as pontuações mais altas, enquanto os estudantes sem esse serviço em casa e com uma opinião insatisfatória em inglês obtiveram as pontuações mais baixas (testes KruskalWallis, $\mathrm{p}=0,0)$. Esses resultados mostram que há novos alunos na UPV que iniciam seus estudos universitários em condições desiguais com os outros. É preocupante que ainda hoje existam estudantes sem acesso à Internet em casa e sem proficiência em inglês satisfatória, pois são condições que reduzem suas chances de sucesso acadêmico. É necessário refletir sobre a desigualdade que afeta esses alunos e as maneiras pelas quais essa situação pode ser melhorada dentro da mesma universidade.

Palavras-chave: divisão digital, compreensão, ensino superior, língua inglesa, leitura.

Fecha Recepción: Mayo 2019

Fecha Aceptación: Noviembre 2019

\section{Introducción}

Es importante detectar los factores de éxito académico de los estudiantes de nuevo ingreso en la universidad con la finalidad de generar propuestas de intervención que ayuden a mejorar sus probabilidades de que finalicen en tiempo y forma sus estudios. Y en ese rubro la comprensión lectora es uno de los factores más influyentes (Gallardo y López, 2019). Si bien hoy los estudiantes pueden acceder a más textos que hace 25 años gracias al Internet, todavía existen estudiantes que en casa sufren la primera brecha digital; aun en países desarrollados existe evidencia de hogares que se pueden clasificar como altamente conectados y hogares no conectados (Robinson y Schulz, 2013). Sin embargo, también existen estudiantes que cuentan con acceso a internet en casa, pero presentan deficiencias lectoras en inglés y en español. Por tanto, se puede mencionar que incluso cuando un estudiante haya superado la primera brecha digital se enfrenta a una segunda, que son las habilidades cognitivas: una de ellas la comprensión de lectura (Büchi, Just y Latzer, 2016). 


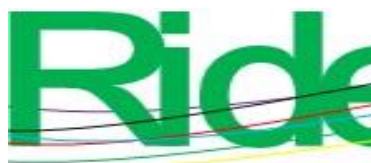

Revista Iberoamericana para la
Investigación y el Desarrollo Educativo
ISSN $2007-7467$

El acceso a la gran variedad de textos en Internet pudiera presentar una dificultad para su lectura dado que leer en esa plataforma pudiera ser más difícil que leer un libro, ya que todos los textos son planos, tienen una forma similar y están en la misma pantalla; además, los textos a los cuales un estudiante puede enfrentarse en Internet pueden ser complejos, que no fueron pensados explícitamente para su nivel de lectura. Así, pues, es importante mencionar que existe una diferencia entre la lectura en formato tradicional frente a la lectura en formato de hipertexto, pues la sobrecarga cognitiva que representa un texto no lineal, tal como lo es el hipertexto, dificulta la comprensión lectora de las personas (Srivastava y Gray, 2012). No está de más enfatizar que esta es una habilidad básica y transversal en la sociedad moderna: todo ciudadano que desee participar en ella debe poseer una buena comprensión de lectura. Llegado a este punto también cabe mencionar que no se debe confundir la comprensión lectora con las competencias básicas de comunicación, ya que aquella es solo una parte del conjunto que conforman estas últimas. Al respecto, Binkley (citado en Organización para la Cooperación y el Desarrollo Económicos [OECD, por sus siglas en inglés], 2019) menciona:

Ya sea un técnico o una persona profesional, el éxito radica en poder comunicarse, compartir y usar información para resolver problemas complejos, para poder adaptarse e innovar en respuesta a nuevas demandas y circunstancias cambiantes, para poder reunir y expandir el poder de la tecnología para crear nuevos conocimientos y ampliar la capacidad humana y la productividad (p. 22).

En la actualidad existe un mayor énfasis en la integración de las tecnologías de la información en la lectura, aunque se mantengan las habilidades básicas de comprensión, interpretación y reflexión en textos individuales (Spiro, DeSchryver, Hagerman, Morsink y Thompson, 2015). Por otra parte, la diferencia entre lectura y lectura digital tiene que ver con el contexto del proceso en el que se realiza (Singer y Alexander, 2017). Elleman y Compton (2017) mencionan que la comprensión lectora se da a partir de una interacción entre el lector, el texto y la tarea, la cual está inmersa en un contexto sociocultural. Bawden (citado en Burin, Coccimiglio, González y Bulla, 2016) comenta que la alfabetización digital está formada de varias habilidades, tales como el conocimiento previo de la información, la comprensión lectora de la información digital y el aprender de forma independiente. 


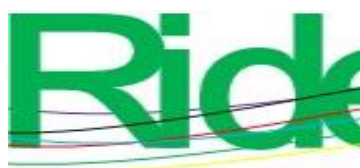

Revista Iberoamericana para la Investigación y el Desarrollo Educativo ISSN $2007-7467$

Por su parte, el idioma inglés, que predomina actualmente en los contenidos en Internet (Correa, Reséndiz, Capello y Recio, 2015), se ha identificado como un claro limitante entre pueblos y sociedades, pero también como un factor esencial en el contexto académico de los estudiantes universitarios (Gómez, Insa y Sanjosé, 2015). En esa tónica, Chávez, Saltos y Saltos (2017) argumentan que el inglés es de vital importancia para que los alumnos universitarios puedan tener buenas oportunidades en un mundo globalizado. Además, añaden que la mayoría de la bibliografía fundamental y las fuentes de información en Internet para las asignaturas universitarias se encuentran en dicha lengua. También citan a Sprachcaffe (2017), quien afirma que aprender un idioma distinto al nativo produce estímulos cerebrales que inciden en el mejoramiento de otras áreas como el razonamiento, la resolución de problemas y la habilidad mental.

En el presente trabajo se estudia la brecha digital y las competencias en idioma inglés en relación con la comprensión lectora, la cual adquiere especial relevancia en la vida académica universitaria. La hipótesis de investigación es que tanto una brecha digital estrecha como un desempeño satisfactorio del idioma inglés están relacionados con efectos positivos en la comprensión lectora en español en los alumnos de la Universidad Politécnica de Victoria (UPV), ubicada en Tamaulipas, México, en específico en aquellos de nuevo ingreso de los años 2015, 2016 y 2017. Para mostrar las diferencias lingüísticas entre grupos de estudiantes que emergieron en este estudio se utilizó una prueba de Kruskal-Wallis con un análisis posterior de Mann-Whitney, siguiendo el estudio de Sánchez y Hernández (2018), quienes utilizan dichas pruebas para evidenciar diferencias lingüísticas entre grupos de estudiantes y evaluar su habilidad para reconocer y manipular morfemas, dado que esta habilidad está fuertemente ligada con el desarrollo de la comprensión lectora del estudiante.

Sin embargo, a diferencia de Sánchez y Hernández (2018), en esta investigación los grupos de estudio se formaron con la finalidad de visualizar diferencias entre estos respecto a la comprensión de lectura en español, el nivel del idioma inglés y el hogar conectado a Internet. En el presente artículo se exponen los resultados de esta investigación, con el objetivo de profundizar en el conocimiento de la comprensión lectora, para lo cual se analizaron los resultados del Examen Nacional de Ingreso a la Educación Superior (ExaniII), diseñado por el Centro Nacional para la Evaluación de la Educación Superior (Ceneval), que presentaron los estudiantes de la población mencionada. El trabajo está organizado de esta manera: primero, se presentan los antecedentes contextuales y trabajos previos. En el 


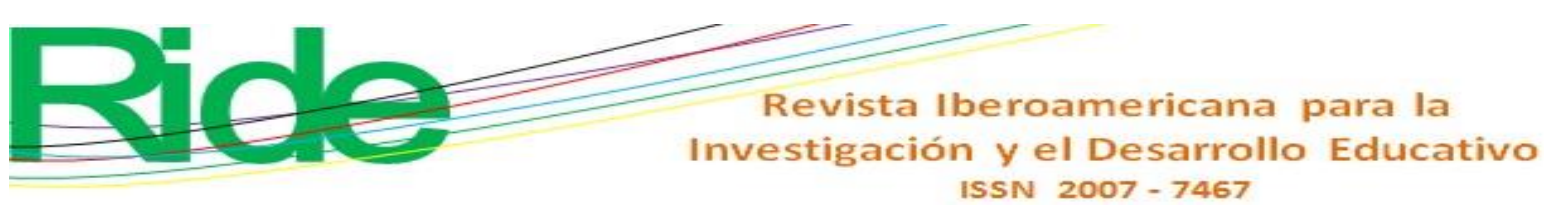

siguiente apartado se explican los detalles de la metodología que se siguió. Luego se presentan los resultados de los análisis, los cuales se discuten en la sección inmediata posterior. Finalmente se abordan las conclusiones.

\section{Brecha digital}

Hoy en día nos encontramos en la era de la globalización. Internet se ha extendido por todo el mundo desde 1998, siguiendo una curva exponencial. De acuerdo con la compañía Internet Advisor (2019), el número de usuarios web alrededor del mundo ha aumentado de 16 millones de personas en el año 1993 a 350 millones en el año 2000; 2267 millones de personas en el año 2010 hasta 4208 millones de personas al 30 junio del 2018. Además, se presume que continúa en expansión. De acuerdo con la Organización de las Naciones Unidas para la Educación, la Ciencia y la Cultura [Unesco] (2005), el efecto de la globalización a finales del siglo XX y comienzos del siglo XXI ha interconectado al mundo en una forma nunca vista en la historia de la humanidad, lo que ha creado nuevos y variados vínculos entre grupos sociales, científicos, tecnológicos y culturales, los cuales están en constante evolución. Sin embargo, a pesar del gran avance tecnológico, aún existen desigualdades sociales; por ejemplo, la denominada primera brecha digital (Ma, Vachon y Cheng, 2019), que se refiere a la diferencia socioeconómica entre personas que tienen acceso a la tecnología digital y quienes no lo tienen. Ma et al. (2019) destacan que el estudio de la primera brecha digital es relevante desde diferentes perspectivas. Por ejemplo, se ha encontrado que las personas que cuentan con acceso a internet tienen mayor poder adquisitivo que aquellas que no lo tienen.

\section{Habilidades de comprensión lectora}

Las habilidades en la comprensión lectora son determinantes para el avance académico de los estudiantes universitarios. Se trata de un proceso complejo desarrollado mediante la lectura frecuente del estudiante y perfeccionado por medio del lenguaje escrito. La comprensión de la lectura se puede clasificar como una capacidad cognitiva; esto es, la persona debe poder convertir la información en conocimiento (Correa et al., 2015). Por lo tanto, entre mayor nivel de comprensión lectora tenga un usuario, mayor provecho podrá obtener de la información existente en Internet. Ahora bien, de acuerdo con el comité de expertos de la OECD (2006), se entiende por competencia lectora lo siguiente: "La capacidad 


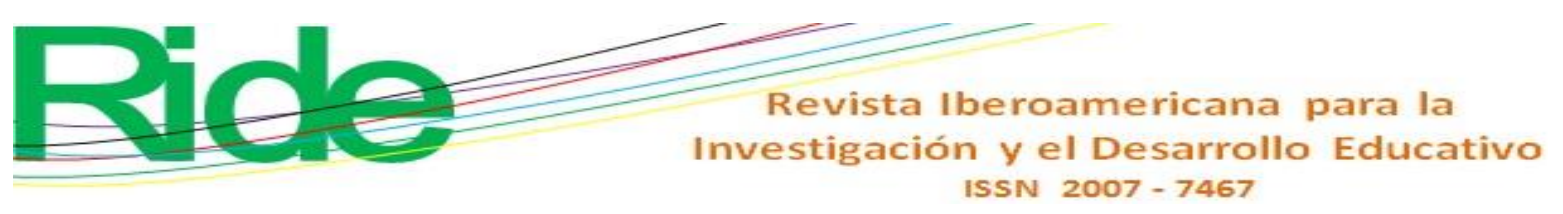

de comprender, utilizar y analizar textos escritos para alcanzar los objetivos personales del lector, desarrollar sus conocimientos y posibilidades y participar en la sociedad" (p. 13). Además, en México, la Secretaría de Educación Pública (SEP) emitió el Acuerdo Número 444 (Diario Oficial de la Federación [DOF], 2008), en el cual se mencionan las competencias que constituyen el marco curricular común del Sistema Nacional de Bachillerato. Y en este acuerdo la comprensión lectora se define así:

La capacidad de los estudiantes de comunicarse efectivamente en el español y en lo esencial en una segunda lengua en diversos contextos, mediante el uso de distintos medios e instrumentos. (...)

Los estudiantes que hayan desarrollado estas competencias podrán leer críticamente y comunicar y argumentar ideas de manera efectiva y con claridad, oralmente y por escrito. Además, usarán las tecnologías de la información y la comunicación de manera crítica para diversos propósitos comunicativos (DOF, 2008, cap. III, art. 7).

El anterior acuerdo establece las bases para el marco de competencias de la comunicación en la educación media superior en México. En resumen, el acuerdo incluye: leer críticamente, comunicarse mediante una segunda lengua y utilizar las tecnologías de la información. Por lo tanto, el acuerdo establece que el estudiante debe dominar una segunda lengua, en este caso el idioma inglés.

Es lógico que este acuerdo fomente comunicarse mediante una segunda lengua, pues la mayor cantidad de nodos y dominios en Internet pertenecen a Estados Unidos y a otros países de habla inglesa (Correa et al., 2015). También es coherente que incluya el dominio del estudiante de las tecnologías de la información y comunicación (TIC), en este caso el uso del Internet, ya que este permite la utilización de ofimática avanzada, consulta de repositorios de datos en línea, tutoriales de instrucción en video, así como la accesibilidad en línea de diferentes escritos literarios y científicos. Además, el acceso a internet produce un cambio positivo en la vida del alumno universitario (Castaño, Duart y Sancho, 2014). Salmerón, García y Vidal (2018), por ejemplo, mencionan que los padres y los maestros deben ser conscientes de la importancia de recomendar lecturas frecuentes de Internet en casa como una forma de mejorar las competencias basadas en la comprensión de lectura en ese espacio. Apoyar otros usos, como las interacciones sociales, no tendrían un impacto positivo en las habilidades de los estudiantes. 


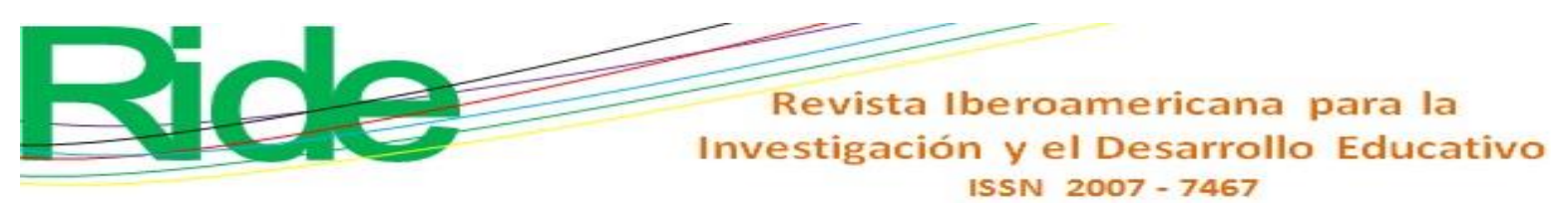

\section{La UPV y el examen Ceneval Exani-II}

El 11 de octubre de 2006, el gobernador del estado de Tamaulipas, por medio de la Secretaría de Educación Estatal, firmó el convenio que daba inicio al proyecto de la UPV. Esta universidad es un organismo público descentralizado del Gobierno del estado de Tamaulipas con personalidad jurídica y patrimonio propios. Para cumplir con la misión de la UPV de formar profesionales competitivos en su área de especialidad, nacional e internacionalmente, es necesario que los estudiantes de nuevo ingreso cumplan con las competencias disciplinares básicas de comunicación. Y para asegurar que el estudiante posee dichas competencias disciplinares, se aplica el examen de admisión Exani-II del Ceneval (2019), que demanda para la comprensión lectora lo puesto a continuación: "Comprender información explícita e implícita en textos informativos, argumentativos y narrativos de mediana complejidad, así como su propósito, características y lenguaje" (p. 12). La comprensión lectora en este examen se evalúa en una escala de 700 a 1300 puntos. Para el idioma inglés, se dictamina únicamente si es satisfactorio o insatisfactorio. Para indagar si el estudiante tiene acceso a internet desde su casa, se le plantea una pregunta que puede ser respondida con "Sí" o "No".

\section{Estudios relacionados}

Hoar (2014) menciona que la mayoría de los criterios de admisión en estudios posteriores a la educación básica requieren buenas habilidades en comprensión de lectura, escritura y matemáticas, las cuales son mencionadas por Shearer y Karanian (2017) como parte de la teoría de inteligencias múltiples. Gómez et al. (2015), por su parte, indican que la adquisición de la lengua inglesa es esencial en el contexto académico de estudiantes universitarios actuales. Por otro lado, Singer y Alexander (2017) realizaron una revisión de la literatura de estudios empíricos que diferencian la comprensión de lectura en medios

digitales comparándola con la comprensión de lectura en medios impresos. Weber y Becker (2017) estudiaron la desigualdad social relacionada con la escuela en el uso del Internet en adolescentes. Es de destacar asimismo el trabajo de Van-Deursen y Van-Dijk (2019), donde se indica que la primera brecha digital sigue siendo un problema en uno de los países más acaudalados y tecnológicamente más desarrollados del mundo, como lo es Holanda. Luna (2015) menciona que en México pueden existir ciudadanos excluidos de la sociedad de la información. En esa misma línea, Ma et al. (2019) hablan de la primera brecha digital, la 


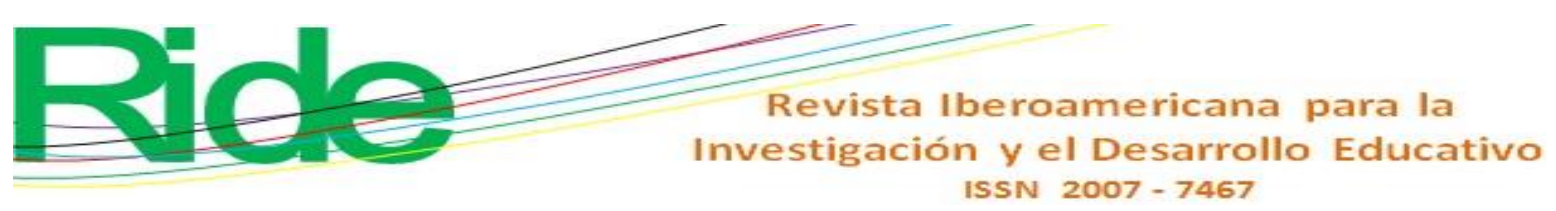

brecha de acceso, que es la diferencia entre personas que tienen acceso a internet y las que no lo tienen; además, se menciona la existencia de la segunda brecha digital introducida por Attewell (2001), que es la diferencia en el uso y habilidades de las personas respecto a las TIC.

Villegas (2017) menciona que el Exani-II del Ceneval (2019) puede ser utilizado como un instrumento de exclusión social. Y Martínez y Herrera (2013) miden el impacto de las TIC mediante el apartado de la escala de autoeficacia en el uso de la computadora del Exani-II, en donde se explora la autopercepción del estudiante para navegar y buscar información en Internet, el uso de un procesador de textos, hoja de cálculo y software para realizar presentaciones. El estudio de Martínez y Herrera (2013) se puede relacionar con la autopercepción de la segunda brecha digital. Otro esfuerzo similar es el de Cortés y Palomar (2008), quienes analizan los resultados de los estudiantes que presentaron el Exani-II con la finalidad de predecir el éxito académico del estudiante a través de su promedio general de preparatoria y un cuestionario de problemas sociales. Un estudio más dentro de esta área es el realizado por Chain, Cruz, Martínez y Jacome (2003), en donde se analiza la trayectoria escolar del alumno agrupando categorías en los criterios de admisión del Exani-II, las cuales se vinculan al éxito académico del estudiante. Algunos de los estudios anteriores tienen como objetivo predecir el éxito académico del estudiante analizando los resultados del Exani-II de Ceneval. Sin embargo, no se enfocan en la primera brecha digital y su asociación con las competencias disciplinares básicas de comunicación (DOF, 2008).

\section{Metodología}

\section{Diseño de investigación}

Se utilizó un diseño cuantitativo, retrospectivo y relacional.

\section{Participantes}

Los participantes fueron el total de alumnos que presentaron el Exani-II del Ceneval de ingreso a la UPV en los años $2015(n=300), 2016(n=281)$ y $2017(n=524)$. 


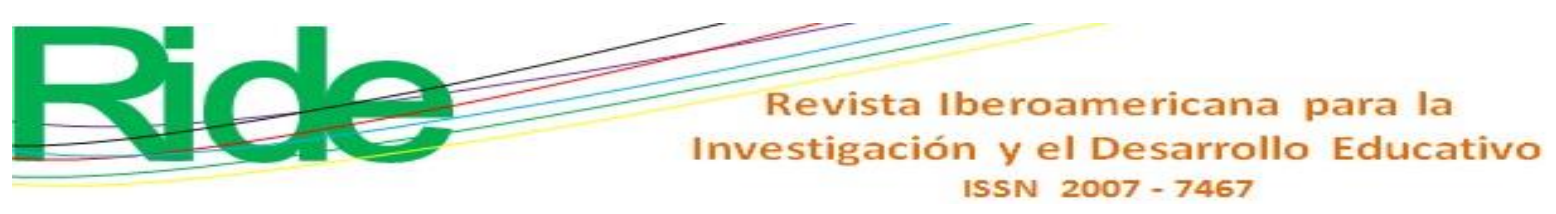

\section{Análisis de datos}

El análisis de los datos de los alumnos de nuevo ingreso en cada año, 2015, 2016 y 2017, se realizó en los paquetes estadísticos SPSS versión 24 y Jamovi versión 1.0.4.0, y se condujo en dos fases: en la primera, se realizó un análisis estadístico con un factor para lo cual se crearon cuatro grupos, como se indica en la tabla 1.

Tabla 1. Clasificación de alumnos de acuerdo con las posibles combinaciones de valores en el dominio del idioma inglés y en su conexión a internet en casa

\begin{tabular}{|c|c|c|}
\hline Grupo & Internet en casa & Dictamen de inglés \\
\hline 1 & Sí & Satisfactorio \\
\hline 2 & No & Satisfactorio \\
\hline 3 & Sí & Insatisfactorio \\
\hline 4 & No & Insatisfactorio \\
\hline
\end{tabular}

Fuente: Elaboración propia

Además, se graficaron las distribuciones de los resultados de la evaluación de la comprensión lectora en español de acuerdo con los años de ingreso. Se realizó una primera inspección visual de las distribuciones. Se observaron, primeramente, similitudes entre los grupos 1 y 2 , y se identificó el factor común, que es el dominio del idioma inglés. Más tarde se observaron similitudes entre los grupos 3 y 4, cuyo factor común era la falta de dominio del idioma inglés (ver figura 1, 2 y 3).

Figura 1. Comprensión lectora en español clasificada de acuerdo con los grupos de la tabla

1 para el año 2015

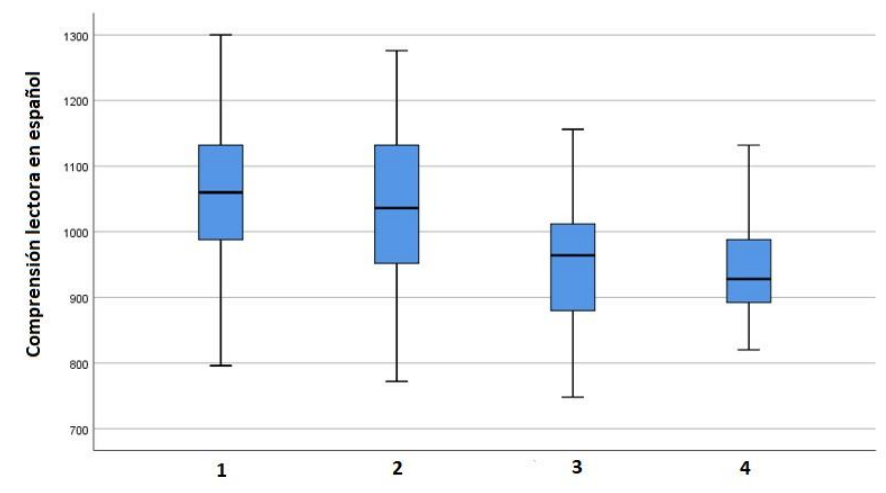

Fuente: Elaboración propia 


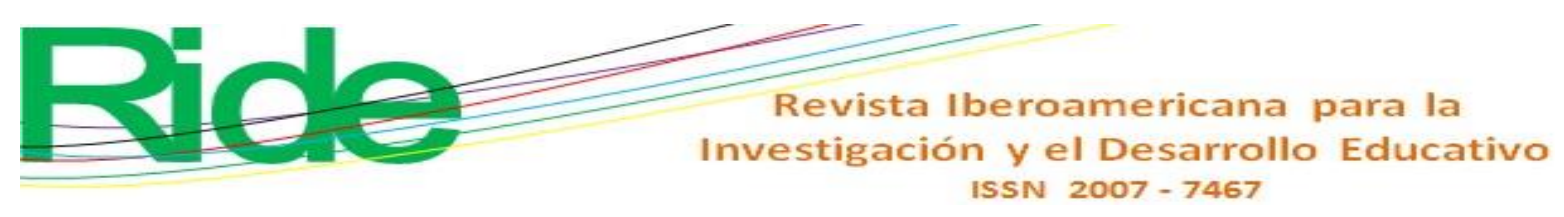

Figura 2. Comprensión lectora en español clasificada de acuerdo con los grupos de la tabla 1 para el año 2016

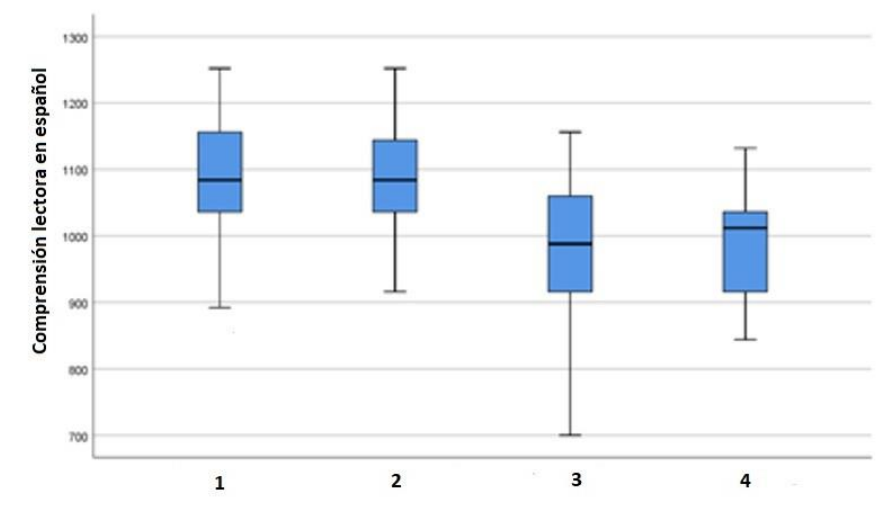

Fuente: Elaboración propia

Figura 3. Comprensión lectora en español clasificada de acuerdo con los grupos de la tabla 1 para el año 2017

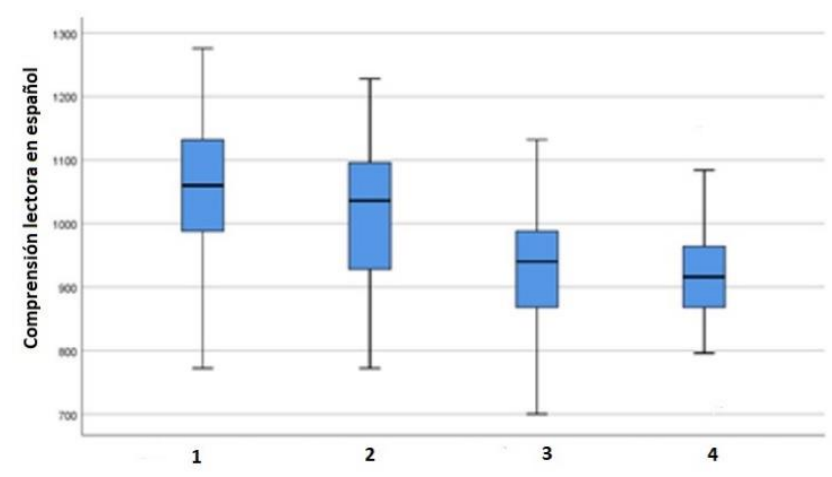

Fuente: Elaboración propia

Se planteó la siguiente hipótesis de investigación para cada año 2015, 2016 y 2017:

- Ha1: Existen diferencias en la comprensión lectora de los alumnos de nuevo ingreso de la UPV entre los grupos de estudio definidos en la tabla 1.

Se realizaron pruebas no paramétricas de Kruskal-Wallis y comparaciones posteriores de Dwass-Steel-Critchlow-Fligner para analizar las diferencias en la puntuación de la comprensión lectora entre los cuatro grupos previamente creados.

En los análisis de la primera fase, de acuerdo con lo presentado en las figuras 1, 2 y 3, se encontraron razones para pensar que el tener el servicio de internet en casa no resultaba tan relevante para la comprensión lectora como el resultado del dictamen de inglés. Por esta 


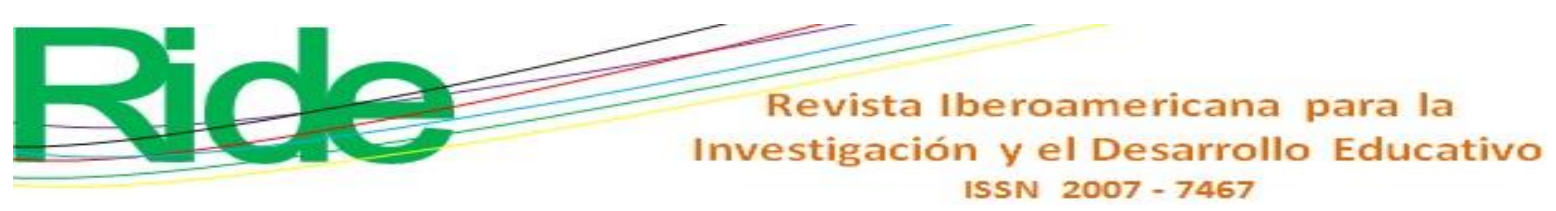

razón se procedió a una segunda fase. Allí se realizó un análisis con dos factores para observar el efecto independiente de cada variable, así como la interacción entre ambas sobre la comprensión lectora. Se hicieron entonces pruebas no paramétricas de Scheirer-Ray-Hare para cada año, en donde se plantearon las siguientes hipótesis de investigación:

Ha2: Existe diferencia en la comprensión lectora entre los alumnos que tienen internet y los que no lo tienen.

- Ha3: Existe diferencia en la comprensión lectora entre los alumnos con dictamen satisfactorio e insatisfactorio en el idioma inglés.

- $\quad$ Ha4: Existe un efecto de interacción entre la brecha digital y el dictamen del idioma inglés, que incide en la comprensión lectora.

\section{Procedimiento}

Primeramente, se recolectaron los datos, los cuales fueron provistos por el departamento de servicios escolares de la UPV. Se obtuvieron tres archivos en formato CSV (separados por comas) con los datos de los resultados del examen Exani-II que presentaron los alumnos de nuevo ingreso en los años 2015, 2016 y 2017. Los archivos fueron importados a SPSS, en donde se realizó una exploración y limpieza de los datos. Se eliminaron las columnas irrelevantes para el objetivo del análisis y se revisaron las condiciones de normalidad (con pruebas de Kolmogorov-Smirnov y Shapiro-Wilk), homocedasticidad (con pruebas de Levene) y forma de las distribuciones (a través de inspección visual). Se determinó que no todos los grupos cumplían con distribución normal e igualdad de varianzas, por lo que se optó por realizar pruebas no paramétricas. De esta manera, los datos fueron importados al software Jamovi, en donde se hizo el análisis unifactorial del resultado de la comprensión lectora para los cuatro grupos de estudio con la finalidad de observar si existía diferencia en la tendencia central de la calificación observada para cada grupo. Este análisis se llevó a cabo para cada año de estudio. También se calcularon los estadísticos descriptivos de media, desviación estándar, mediana y rango intercuartil. Para el análisis con un factor se realizaron pruebas de Kruskal-Wallis con comparaciones posteriores de Dwass-SteelCritchlow-Fligner. Posteriormente, también en Jamovi, se llevó a cabo un análisis con dos factores en el que se observó cada variable de manera independiente y la relación entre ambas, para lo cual se realizaron pruebas de Scheirer-Ray-Hare a través del módulo Rj Editor. 


\section{Resultados}

\section{Fase 1. Análisis con un factor}

Los resultados de la prueba Kruskal-Wallis para el año 2015 fueron los siguientes: $H$ $=42.17, g l=3, p=0, \varepsilon^{2}=0.141$. Para obtener las diferencias estadísticamente relevantes entre estos cuatro grupos, se realizaron comparaciones posteriores con la prueba DwassSteel-Critchlow-Fligner. Los resultados son mostrados en la tabla 2. Mientras que en la tabla 3 son mostrados los valores descriptivos de los datos del año 2015.

Tabla 2. Comparaciones de Dwass-Steel-Critchlow-Fligner para el año 2015

\begin{tabular}{|c|c|c|c|}
\hline \multicolumn{2}{|c|}{ Comparación entre los grupos } & W & P \\
\hline 1 & 2 & -1.016 & 0.890 \\
\hline 1 & 3 & -7.057 & $<0.001$ \\
\hline 1 & 4 & -6.974 & $<0.001$ \\
\hline 2 & 3 & -5.299 & 0.001 \\
\hline 2 & 4 & -5.368 & $<0.001$ \\
\hline 3 & 4 & -0.834 & 0.935 \\
\hline
\end{tabular}

Fuente: Elaboración propia

Tabla 3. Valores descriptivos de cada grupo en el año 2015

\begin{tabular}{|c|c|c|c|c|c|c|c|}
\hline Grupo & $\begin{array}{c}\text { Internet } \\
\text { en casa }\end{array}$ & $\begin{array}{c}\text { Dictamen de } \\
\text { inglés }\end{array}$ & $\boldsymbol{N}$ & Media & $\begin{array}{c}\text { Desv. } \\
\text { Std }\end{array}$ & Mediana & $\begin{array}{c}\text { Rango } \\
\text { intercuartil }\end{array}$ \\
\hline 1 & Sí & Satisfactorio & 156 & 1049.23 & 110.31 & 1060 & 144 \\
\hline 2 & No & Satisfactorio & 75 & 1037.28 & 118.62 & 1036 & 192 \\
\hline 3 & Sí & Insatisfactorio & 39 & 949.23 & 94.34 & 964 & 144 \\
\hline 4 & No & Insatisfactorio & 30 & 940.80 & 89.01 & 928 & 102 \\
\hline
\end{tabular}

Fuente: Elaboración propia

Los resultados de la prueba Kruskal-Wallis para el año 2016 fueron los siguientes: $H$ $=49.28, g l=3, p=0, \varepsilon^{2}=0.176$. Las diferencias estadísticamente relevantes entre estos cuatro grupos se obtuvieron a través de comparaciones posteriores con la prueba DwassSteel-Critchlow-Fligner. Los resultados se muestran en la tabla 4. Los valores descriptivos para el año 2016 se muestran en la tabla 5. 


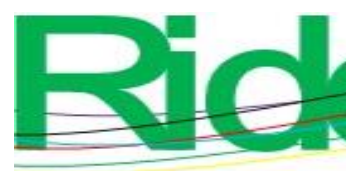

Revista Iberoamericana para la Investigación y el Desarrollo Educativo ISSN $2007-7467$

Tabla 4. Comparaciones de Dwass-Steel-Critchlow-Fligner para el año 2016

\begin{tabular}{|c|c|c|c|}
\hline \multicolumn{2}{|c|}{ Comparación entre los grupos } & W & P \\
\hline 1 & 2 & -0.215 & 0.999 \\
\hline 1 & 3 & -7.983 & $<0.001$ \\
\hline 1 & 4 & -6.654 & $<0.001$ \\
\hline 2 & 3 & -6.252 & $<0.001$ \\
\hline 2 & 4 & -5.787 & $<0.001$ \\
\hline 3 & 4 & -0.758 & 0.950 \\
\hline
\end{tabular}

Fuente: Elaboración propia

Tabla 5. Valores descriptivos de cada grupo en el año 2016

\begin{tabular}{|c|c|c|c|c|c|c|c|}
\hline Grupo & $\begin{array}{c}\text { Internet } \\
\text { en casa }\end{array}$ & $\begin{array}{c}\text { Dictamen de } \\
\text { inglés }\end{array}$ & $\boldsymbol{N}$ & Media & $\begin{array}{c}\text { Desv. } \\
\text { Std }\end{array}$ & Mediana & $\begin{array}{c}\text { Rango } \\
\text { intercuartil }\end{array}$ \\
\hline 1 & Sí & Satisfactorio & 161 & 1090.86 & 84.14 & 1084 & 120 \\
\hline 2 & No & Satisfactorio & 55 & 1086.62 & 96.18 & 1084 & 120 \\
\hline 3 & Sí & Insatisfactorio & 46 & 990.61 & 100.58 & 988 & 150 \\
\hline 4 & No & Insatisfactorio & 19 & 977.89 & 84.78 & 1012 & 168 \\
\hline
\end{tabular}

Fuente: Elaboración propia

Los resultados de la prueba Kruskal-Wallis para el año 2017 fueron los siguientes: $H$ $=121.60, g l=3, p=0, \varepsilon^{2}=0.233$. Para obtener las diferencias estadísticamente relevantes entre estos cuatro grupos, se realizaron comparaciones posteriores con la prueba DwassSteel-Critchlow-Fligner. Los resultados se muestran en la tabla 6. Los valores descriptivos para el año 2017 se muestran en la tabla 7. 


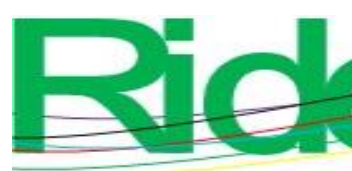

Revista Iberoamericana para la Investigación y el Desarrollo Educativo ISSN 2007 - 7467

Tabla 6. Comparaciones de Dwass-Steel-Critchlow-Fligner para el año 2017

\begin{tabular}{|c|c|c|c|}
\hline \multicolumn{2}{|c|}{ Comparación entre los grupos } & W & P \\
\hline 1 & 2 & -3.065 & 0.133 \\
\hline 1 & 3 & -12.388 & $<0.001$ \\
\hline 1 & 4 & -11.829 & $<0.001$ \\
\hline 2 & 3 & -6.809 & $<0.001$ \\
\hline 2 & 4 & -6.922 & $<0.001$ \\
\hline 3 & 4 & -0.740 & 0.954 \\
\hline
\end{tabular}

Fuente: Elaboración propia

Tabla 7. Valores descriptivos de cada grupo en el año 2017

\begin{tabular}{|l|l|l|l|l|l|l|l|}
\hline Grupo & $\begin{array}{c}\text { Internet } \\
\text { en casa }\end{array}$ & $\begin{array}{c}\text { Dictamen de } \\
\text { inglés }\end{array}$ & N & Media & $\begin{array}{c}\text { Desv. } \\
\text { Std }\end{array}$ & Mediana & $\begin{array}{c}\text { Rango } \\
\text { intercuartil }\end{array}$ \\
\hline 1 & Sí & Satisfactorio & 297 & 1045.86 & 107.16 & 1060 & 144 \\
\hline 2 & No & Satisfactorio & 75 & 1012.96 & 112.04 & 1036 & 192 \\
\hline 3 & Sí & Insatisfactorio & 87 & 927.31 & 86.36 & 940 & 120 \\
\hline 4 & No & Insatisfactorio & 65 & 919.69 & 78.60 & 916 & 96 \\
\hline
\end{tabular}

Fuente: Elaboración propia

\section{Fase 2. Análisis con dos factores}

Para el año 2015, la prueba de Scheirer-Ray-Hare permitió obtener los siguientes valores: para Ha1: $H=2.33, p=0.12$; para Ha2: $H=39.84, p=0$; para Ha3: $H=0.02, p=$ 0.96. De esta manera, se pudo determinar que para el año 2015 solamente el dictamen del idioma inglés resultó significativo para la comprensión lectora. En la tabla 8 se puede observar que los alumnos con dictamen satisfactorio tuvieron puntuaciones más altas $($ mediana $=1060$, rango intercuartil $=168)$ que los alumnos con dictamen no satisfactorio $($ mediana $=940$, rango intercuartil $=132)$. 


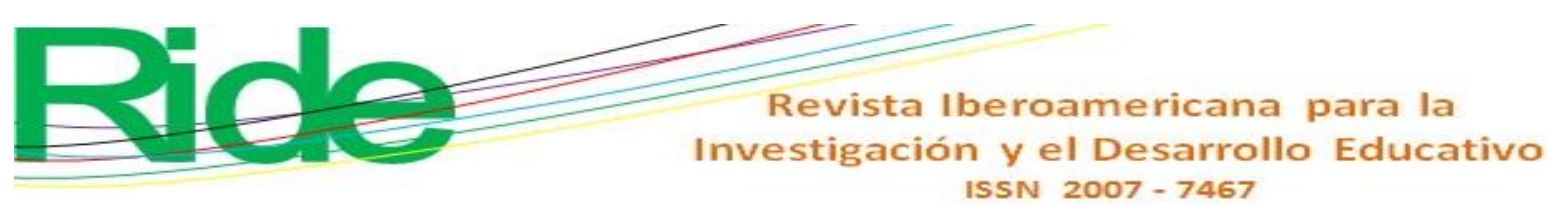

rango intercuartil $=144)$. Los resultados estadísticos descriptivos que se obtuvieron para el año 2017 se muestran en la tabla 10.

Tabla 10. Estadísticos descriptivos de los datos analizados con la prueba de Scheirer-RayHare para el año 2017

\begin{tabular}{|c|c|c|c|}
\hline & Con internet & Sin Internet & $\begin{array}{c}\text { Totales } \\
\text { dictamen de } \\
\text { inglés } \\
\end{array}$ \\
\hline $\begin{array}{c}\text { Dictamen } \\
\text { satisfactorio en inglés }\end{array}$ & $\begin{array}{l}n=297 \\
\text { media }=1045.86 \\
\text { Desv. std. }=107.16 \\
\text { Mediana }=1060 \\
\text { Rango int. }=144\end{array}$ & $\begin{array}{l}n=75 \\
\text { Media }=1012.96 \\
\text { Desv. std. }=112.04 \\
\text { Mediana }=1036 \\
\text { Rango int. }=192\end{array}$ & $\begin{array}{l}n=372 \\
\text { Media } \\
1039.23 \\
\text { Desv. std. = } \\
108.82 \\
\text { Mediana = } \\
1036 \\
\text { Rango int. = } \\
144\end{array}$ \\
\hline $\begin{array}{c}\text { Dictamen } \\
\text { insatisfactorio en } \\
\text { inglés }\end{array}$ & $\begin{array}{l}n=87 \\
\text { Media }=927.31 \\
\text { Desv. std. }=86.36 \\
\text { Mediana }=940 \\
\text { Rango int. }=120\end{array}$ & $\begin{array}{l}n=65 \\
\text { Media }=919.69 \\
\text { Desv. std. }=78.60 \\
\text { Mediana }=916 \\
\text { Rango int. }=96\end{array}$ & $\begin{array}{l}n=152 \\
\text { Media = 924.05 } \\
\text { Desv. std. = } \\
82.95 \\
\text { Mediana = 916 } \\
\text { Rango int. = } \\
120\end{array}$ \\
\hline $\begin{array}{c}\text { Totales Internet en } \\
\text { casa }\end{array}$ & $\begin{array}{l}n=384 \\
\text { Media }=1019 \\
\text { Desv. std. }=114.10 \\
\text { Mediana }=1012 \\
\text { Rango int. }=168\end{array}$ & $\begin{array}{l}n=140 \\
\text { Media }=969.66 \\
\text { Desv. std. }=108.20 \\
\text { Mediana }=952 \\
\text { Rango int. }=144\end{array}$ & \\
\hline
\end{tabular}

Fuente: Elaboración propia

\section{Discusión}

\section{Interpretación del análisis con un factor}

Los siguientes hallazgos fueron comunes para los tres años analizados: 2015, 2016 y 2017. Se encontró que existen diferencias estadísticas significativas en la comprensión lectora de los estudiantes según el grupo de estudio al que pertenecen. Se observó que los alumnos que tienen internet en casa y que obtuvieron un dictamen satisfactorio en el idioma inglés obtuvieron las puntuaciones más altas, mientras que los alumnos sin internet en casa y con un dictamen insatisfactorio en inglés tuvieron las puntuaciones más bajas. Los alumnos 


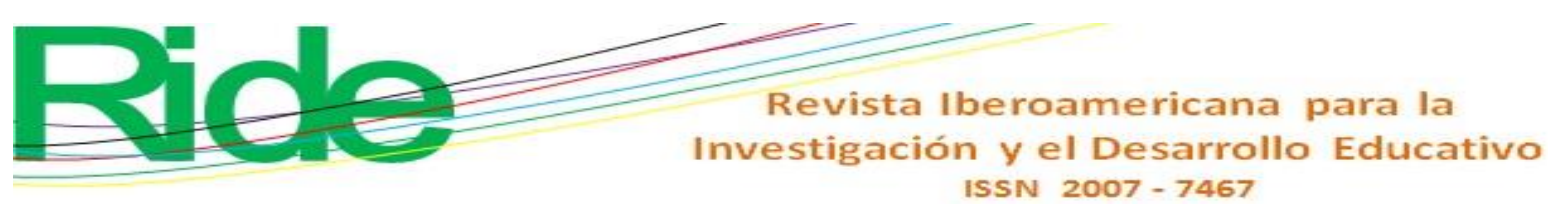

que contaron con solo uno de los dos factores estudiados se mantuvieron en una zona de puntuaciones aritméticas intermedias.

En las pruebas posteriores se observó que todas las diferencias entre los grupos fueron estadísticamente significativas con excepción de las comparaciones entre los grupos 1 y 2 , así como entre los grupos 3 y 4. En ambos casos, la variable constante era el dictamen de inglés y la variable con cambio era la que medía la primera brecha digital. De esta manera, había motivo para pensar que el tener internet en casa no resultaba tan relevante para la comprensión lectora como el resultado del dictamen de inglés. Por esta razón se decidió realizar un análisis con dos factores para observar el efecto independiente de cada variable, así como la interacción entre ambas sobre la comprensión lectora.

\section{Interpretación del análisis con dos factores}

El análisis con dos factores mostró que, en los tres años analizados, el dictamen del idioma inglés fue la variable con efecto significativo en la comprensión lectora. Solamente en el año 2017 también la variable indicadora de la primera brecha digital resultó significativa. De esta manera, en los años 2015, 2016 y 2017, los alumnos con un dictamen favorable en idioma inglés obtuvieron puntuaciones más altas en comprensión lectora. Por otra parte, aunque también en los tres años los alumnos con internet en casa obtuvieron el puntaje más alto en comprensión lectora, esta relación solamente resultó estadísticamente significativa en el año 2017.

\section{Implicación de los resultados}

Los resultados dejan en evidencia que un segmento de estudiantes de nuevo ingreso a la UPV inicia sus estudios universitarios en desigualdad de condiciones con el resto. La falta de acceso al Internet desde el hogar y el dominio insatisfactorio del idioma inglés están relacionados con la comprensión lectora, la cual es una competencia transversal requerida y necesaria en la universidad. Los resultados invitan a tomar medidas para subsanar las deficiencias detectadas y apoyar a los alumnos en sus metas académicas. 


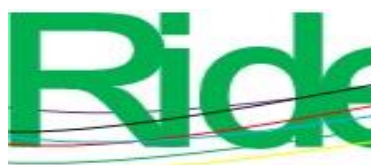

Revista Iberoamericana para la Investigación y el Desarrollo Educativo ISSN $2007-7467$

\section{Comparación de los resultados obtenidos con la literatura analizada}

Los hallazgos de esta investigación son concordantes con el trabajo de Castaño et al. (2014), pues indican que una reducida brecha digital está relacionada con condiciones favorables en la vida académica de los estudiantes. También coinciden con la perspectiva de Ma et al. (2019), quienes expone que el estudio de la brecha digital es relevante desde diversos ángulos. En la presente investigación se encontró que los alumnos con acceso a Internet en casa tienden a obtener mayores puntuaciones en comprensión lectora. Asimismo, los resultados se encuentran en la misma línea de las sugerencias de Salmerón et al. (2018), quienes relacionan positivamente las actividades de Internet en la casa - como lecturas provenientes de ese espacio digital — con la comprensión de lectura. Finalmente, los autores de este trabajo también destacan, tal como lo hacen Martínez y Herrera (2013), que el análisis de los resultados del examen Exani-II puede utilizarse como medio de investigación y diagnóstico de las condiciones que caracterizan a los estudiantes de nuevo ingreso.

\section{Limitaciones del estudio}

El estudio se circunscribió a Cd. Victoria, Tamaulipas, México, concretamente a los alumnos de nuevo ingreso a la UPV. Se tomaron en cuenta únicamente los datos de los alumnos de nuevo ingreso en los años 2015, 2016, 2017, que fueron provistos por el departamento de servicios escolares de la UPV.

\section{Conclusiones}

La investigación presentada en este artículo confirmó la existencia de la primera brecha digital entre los alumnos de nuevo ingreso a la UPV, quienes son parte de un hogar sin servicio a internet. Otro hallazgo develó la existencia de alumnos que no poseen un nivel satisfactorio en el dominio del idioma inglés y además poseen bajo nivel de comprensión lectora en español. Se demostró que estos dos factores en conjunto crean una desventaja académica en comparación con los alumnos que sí forman parte de un hogar con internet y poseen un nivel adecuado de inglés. Aunado a ello, se mostró la importancia de estudiar y reducir la brecha digital, así como de promover en los estudiantes habilidades de comprensión lectora en inglés y español. 


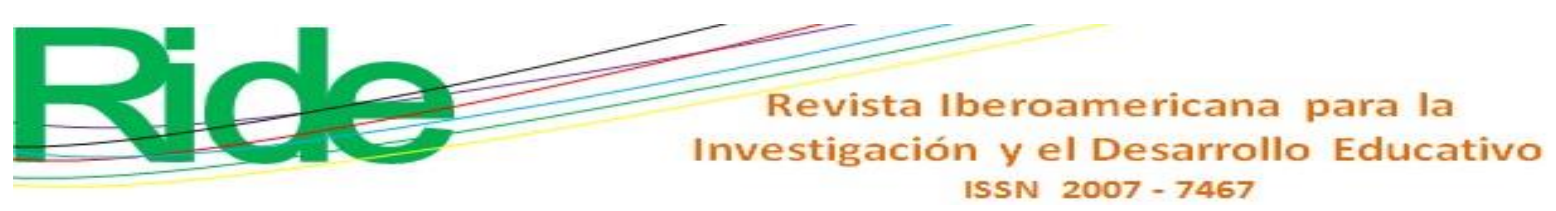

Esto conduce a reflexionar sobre la desigualdad de condiciones de algunos alumnos y la manera en la que se puede contribuir a mejorar esta situación desde el interior de la misma universidad. En este sentido, la creación de programas de apoyo e inclusión puede ser una alternativa viable en la que se debe involucrar a los estudiantes desde antes de su ingreso oficial a la universidad. Este es un reto que se debe enfrentar, aunado al proceso de diseño, creación y mantenimiento de iniciativas para atender a estudiantes en situaciones académicas vulnerables.

Este estudio representa un primer acercamiento para entender la brecha digital en un contexto de realidad social compleja como el de la UPV. Los hallazgos permitieron entender la relación entre algunos factores que pueden incidir en el desempeño académico de los estudiantes. Se plantean, como trabajos futuros, estudios que identifiquen causalidades entre las variables estudiadas, para lo cual se podría diseñar y aplicar un instrumento de recolección de datos distinto al Exani-II del Ceneval. 


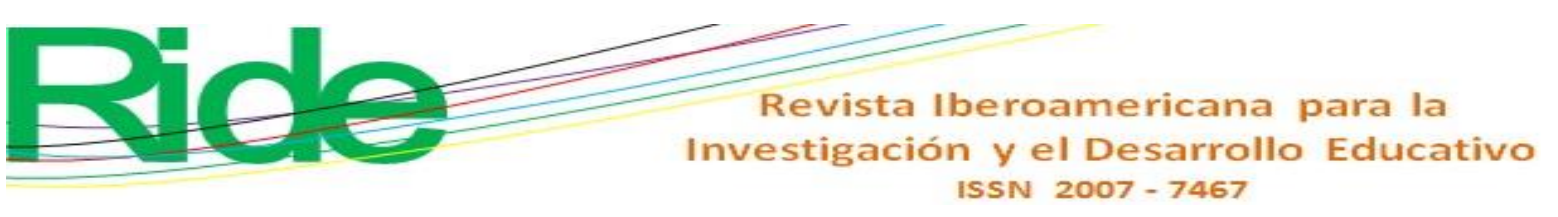

Diario Oficial de la Federación [DOF]. (21 de octubre de 2008). Acuerdo No. 444 de la Secretaría de Educación Pública. Diario Oficial de la Federación. Recuperado de http://dof.gob.mx/nota_detalle.php?codigo=5064951\&fecha=21/10/2008.

Elleman, A. M. and Compton, D. L. (2017). Beyond comprehension strategy instruction: What's next? Language, Speech, and Hearing Services in Schools, 48, 84-91.

Gómez, A., Insa, J. R. and Sanjosé, V. (2015). Postgraduates' Science Comprehension Monitoring in EFL. Bordón. Revista de Pedagogía, 67(4), 27-42. Retrieved from https://doi.org/10.13042/Bordon.2015.67404.

Hoar, R. (2014). Generally Educated in the 21st Century: The Importance Of Computer Literacy In An Undergraduate Curriculum. Paper presented at the 14th Western Canadian Conference on Computing Education. Richmond, May 2-3, 2014.

Internet Advisor (2019). Key Internet Statistics in 2019. Internet Advisor. Retrieved from https://www.internetadvisor.com/key-internet-statistics.

Gallardo, S. V. y López, E. G. (2019). Conocimiento y uso de estrategias de comprensión lectora en estudiantes de psicología. Revista Electrónica de Psicología Iztacala, 22(2), 2066-2086.

Luna, I. (2015). La otra brecha digital. La sociedad de la información y el conocimiento. Ciudad de México, México: UNAM. Recuperado de http://www.librosoa.unam.mx/xmlui/handle/123456789/444.

Ma, J. K., Vachon, T. E. and Cheng, S. (2019). National Income, Political Freedom, and Investments in R\&D. Social Indicators Research, 144(1), 133-166. Retrieved from http://link.springer.com/10.1007/s11205-018-2030-0.

Martínez, J. A. and Herrera, M. (2013). Psychometric Properties of the Computer SelfEfficacy Scale for EXANI-II. Revista electrónica de investigación educativa, 16(2), 68-80. Retrieved from http://www.scielo.org.mx/scielo.php?pid=S1607$40412014000200005 \&$ script=sci_arttext\&tlng=en.

Organización de las Naciones Unidas para la Educación, la Ciencia y la Cultura [Unesco]. (2005). Hacia las sociedades del conocimiento: informe mundial de la UNESCO. París, Francia: Organización de las Naciones Unidas para la Educación, la Ciencia y la Cultura. Recuperado de http://www.lacult.unesco.org/docc/2005_hacia_las_soc_conocimiento.pdf. 


\section{Revista Iberoamericana para la Investigación y el Desarrollo Educativo ISSN $2007-7467$}

Villegas, R. (2017). ¿Es el EXANI II un predictor del logro académico o un factor de exclusión social? Educación y ciencia, 6(48). Recuperado de http://www.educacionyciencia.org/index.php/educacionyciencia/article/view/444.

Weber, M. and Becker, B. (2019). Browsing the Web for School: Social Inequality in Adolescents' School-Related Use of the Internet. SAGE open, 9(2). Retrieved from https://doi.org/10.1177/2158244019859955.

\begin{tabular}{|l|l|}
\hline Rol de Contribución & Autor (es) \\
\hline Conceptualización & Hiram Herrera \\
\hline Metodología & Ramón Roque \\
\hline
\end{tabular}




\begin{tabular}{|c|c|}
\hline 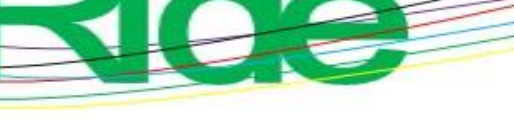 & $\begin{array}{l}\text { Revista Iberoamericana para la } \\
\text { Investigación y el Desarrollo Educativo } \\
\text { ISSN } 2007-7467\end{array}$ \\
\hline Software & Ramón Roque \\
\hline Validación & Hiram Herrera \\
\hline Análisis Formal & Ramón Roque \\
\hline Investigación & Hiram Herrera \\
\hline Recursos & Hiram Herrera \\
\hline Curación de datos & Ramón Roque \\
\hline $\begin{array}{l}\text { Escritura - Preparación del borrador } \\
\text { original }\end{array}$ & Ramón Roque \\
\hline Escritura - Revisión y edición & Hiram Herrera \\
\hline Visualización & Hiram Herrera \\
\hline Supervisión & Ramón Roque \\
\hline Administración de Proyectos & Hiram Herrera \\
\hline Adquisición de fondos & Hiram Herrera \\
\hline
\end{tabular}

